Mário Mendes de Oliveira Zimmermann

\title{
LuaPS - Lua Publish-Subscribe
}

Dissertação apresentada como requisito parcial para obtenção do grau de Mestre pelo Programa de Pós-graduação em Informática do Departamento de Informática da PUC-Rio

Orientador: Prof. Noemi de La Rocque Rodriguez 


\section{Mário Mendes de Oliveira Zimmermann}

\section{LuaPS - Lua Publish-Subscribe}

Dissertação apresentada como requisito parcial para obtenção do grau de Mestre pelo Programa de Pós-graduação em Informática do Departamento de Informática do Centro Técnico Científico da PUC-Rio. Aprovada pela Comissão Examinadora abaixo assinada.

Prof. Noemi de La Rocque Rodriguez

Orientador

Departamento de Informática — PUC-Rio

Prof. Markus Endler

Departamento de Informática - PUC-Rio

Prof. Renato Fontoura de Gusmão Cerqueira

Departamento de Informática - PUC-Rio

Prof. José Eugênio Leal

Coordenador Setorial do Centro Técnico Científico - PUC-Rio 
Todos os direitos reservados. É proibida a reprodução total ou parcial do trabalho sem autorização da universidade, do autor e do orientador.

Mário Mendes de Oliveira Zimmermann

Graduou-se em Engenharia da Computação na PUC-Rio. Trabalhou no desenvolvimento de aplicações para dispositivos móveis. Durante o mestrado, desenvolveu um sistema publishsubscribe que define uma arquitetura focada em generalidade, flexibilidade e extensibilidade.

Ficha Catalográfica

Zimmermann, Mário

LuaPS - Lua Publish-Subscribe / Mário Mendes de Oliveira Zimmermann; orientador: Noemi de La Rocque Rodriguez. - Rio de Janeiro : PUC-Rio, Departamento de Informática, 2006.

v., 75 f: il. ; $29,7 \mathrm{~cm}$

1. Dissertação (mestrado) - Pontifícia Universidade Católica do Rio de Janeiro, Departamento de Informática.

Inclui referências bibliográficas.

1. Informática - Tese. 2. Publish-Subscribe. 3. Lua. 4. Tabela Hash Distribuída. 5. Flexibilidade. 6. Extensibilidade. I. Rodriguez, Noemi. II. Pontifícia Universidade Católica do Rio de Janeiro. Departamento de Informática. III. Título. 


\section{Agradecimentos}

À minha orientadora, Noemi Rodriguez, sempre disponível para ajudar e sem a qual o estudo não seria possível.

À CAPES, à FAPERJ e à PUC-Rio pelos auxílios concedidos.

À minha família, cuja presença e apoio foram fundamentais para o meu desenvolvimento pessoal. 


\section{Resumo}

Zimmermann, Mário; Rodriguez, Noemi. LuaPS - Lua Publish-

Subscribe. Rio de Janeiro, 2006. 75p. Dissertação de Mestrado Departamento de Informática, Pontifícia Universidade Católica do Rio de Janeiro.

Sistemas publish-subscribe são definidos por seu modelo básico de comunicação. No entanto, a maior parte dos sistemas publish-subscribe existentes incorpora outros mecanismos em sua implementação. Este trabalho busca um melhor entendimento de sistemas publish-subscribe, definindo uma arquitetura onde diferentes camadas agrupam decisões e construções relacionadas. Baseado nesta arquitetura, descrevemos um sistema desenvolvido em Lua que utiliza uma tabela hash distribuída como base. O sistema se diferencia dos sistemas publish-subscribe monolíticos e tem como foco generalidade, flexibilidade e extensibilidade.

\section{Palavras-chave}

Publish-Subscribe. Lua. Tabela Hash Distribuída. Flexibilidade. Extensibilidade. 


\section{Abstract}

Zimmermann, Mário; Rodriguez, Noemi. LuaPS - Lua PublishSubscribe. Rio de Janeiro, 2006. 75p. MsC Thesis — Department of Informática, Pontifícia Universidade Católica do Rio de Janeiro.

Publish-subscribe systems are defined by its communication model. However, most of the existent publish-subscribe systems incorporate other mechanisms in their implementation. This work seeks a better understanding of publish-subscribe systems, defining an architecture where different layers group related decisions and constructions. Based on this architecture, we describe a system developed in Lua that uses a distributed hash table as its base. The system differs in its architecture from monolithic publish-subscribe systems and focus on generality, flexibility and extensibility.

\section{Keywords}

Publish-Subscribe. Lua. Distributed Hash Table. Flexibility. Extensibility. 


\section{Sumário}

1 Introdução $\quad 9$

1.1 Publish-Subscribe $\quad 9$

$\begin{array}{lll}1.2 & \text { Objetivos e Organização } & 10\end{array}$

2 Sistemas Utilizados $\quad 13$

2.1 Lua e ALua - Orientação a Eventos 13

2.2 Lua Pastry - Tabela Hash Distribuída (DHT) 19

3 Arquitetura do Sistema $\quad 24$

$\begin{array}{ll}3.1 & \text { Rede LuaPS } \\ 3.2 & 24\end{array}$

$\begin{array}{ll}3.2 & \text { Divisão em Camadas }\end{array}$

4 Camada Publish-Subscribe $\quad 32$

4.1 Gerência sobre Tópicos e Inscrições 34

4.2 Função de Publicação 36

4.3 Entradas e Saídas de Nós na Rede Publish-Subscribe 39

4.4 Execução e Buferização de Comandos 42

5 Camada de Serviços $\quad 44$

5.1 Integração com as Outras Camadas 45

5.2 Acesso a Notificações Antigas - Histórias 47

5.3 Gateway Publish-Subscribe $\quad 50$

5.4 Tolerância a falhas 56

5.5 Combinando Módulos de Serviço 61

5.6 Arquitetutra e Extensibilidade 63

6 Camada de Aplicação $\quad 65$

$\begin{array}{lll}6.1 & \text { Metodologias de Teste } & 65\end{array}$

6.2 Teste das Funcionalidades 66

$\begin{array}{lll}6.3 & \text { Teste de Carga } & 67\end{array}$

7 Considerações Finais $\quad \mathbf{7 0}$

$\begin{array}{lll}7.1 & \text { Trabalhos Relacionados } & 70\end{array}$

$\begin{array}{ll}7.2 \text { Conclusão } & 71\end{array}$

$\begin{array}{ll}\text { Referências Bibliográficas } & 73\end{array}$ 


\section{Lista de figuras}

2.1 Modelo de Comunicação 14

2.2 Console de Interação com o Usuário 17

2.3 Comunicação Console-Processo 18

2.4 Tabela de Roteamento para o Nó com ID 10233102

3.1 Arquitetura Heterogênea 24

3.2 Arquitetura Homogênea 26

3.3 Camadas do Projeto LuaPS 28

3.4 Módulos da Camada de Serviços 30

4.1 Estrutura utilizada na Gerência dos Tópicos 35

4.2 Função de Publicação Simples 37

4.3 Função de Publicação utilizada como Filtro 39

4.4 Mudança na Gerência dos Tópicos com a Entrada de Nós na Rede 39

4.5 Protocolo para Entrada de Nós na Rede 40

4.6 Protocolo para Saída de Nós da Rede 41

4.7 Estrutura utilizada na Gerência da Execução dos Comandos 43

5.1 Integração Clássica 45

5.2 Integração Múltipla $\quad 46$

5.3 Integração Híbrida 47

5.4 Estrutura de Armazenamento das Histórias 49

5.5 Arquitetura de Comunicação com Utilização do Gateway 52

5.6 Tabela de Mensagens Armazenadas para Reenvio 54

5.7 Estrutura de Backup 58

5.8 Processo de Restauração do Backup 60

5.9 Aplicação com um Módulo de Serviço 62

5.10 Aplicação com dois Módulos de Serviço Combinados 63

6.1 Resultados do Teste de Carga $\quad 69$ 